\title{
Relationship between the Nonexponentiality of Relaxation and Relaxation Time in the Problem of Glass Transition
}

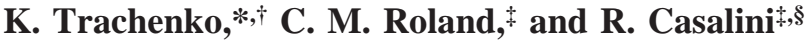 \\ Department of Earth Sciences, University of Cambridge, Cambridge CB2 3EQ, U.K., Naval Research \\ Laboratory, Washington, District of Columbia 20375-5342, and George Mason University, \\ Fairfax, Virginia 22030
}

Received: January 14, 2008; In Final Form: February 20, 2008

\begin{abstract}
By analyzing the experimental data for various glass-forming liquids and polymers, we find that the nonexponentiality, $\beta$, and the relaxation time, $\tau$, are commonly related: $\log (\tau)$ is an approximately linear function of $1 / \beta$, followed in most cases by a crossover to a higher linear slope. We rationalize this relationship in the recently developed elastic approach to the glass transition. The key to the observed common relationship between $\beta$ and $\tau$ is that the two quantities are governed by the same parameter, the liquid elasticity length, $d_{\mathrm{el}}$. The increase of $d_{\mathrm{el}}$ on lowering temperature increases $\tau$ and decreases $\beta$, resulting in the observed common relationship between $\beta$ and $\tau$. In this picture, we also discuss the crossovers of $\beta$ and $\tau$ at low temperature.
\end{abstract}

\section{Introduction}

Cooling a liquid to obtain a glass may seem a familiar and conceptually simple process, yet its theoretical description remains elusive. Such a description, as widely perceived, should provide a consistent theory for the two main properties that a liquid acquires in the glass transformation range: nonexponential relaxation and super-Arrhenius temperature dependence of relaxation time. ${ }^{1}$

At high temperature, a liquid under external perturbation relaxes exponentially fast: a relaxing quantity $q(t)$ decays as $\exp (-(t / \tau))$, where $\tau$ is associated with the transition over a single activation barrier. This is known as Debye relaxation. On lowering the temperature, relaxation changes markedly, and is described by a stretched-exponential function, $q(t) \propto \exp (-$ $\left.(t / \tau)^{\beta}\right)$, where $0<\beta \leq 1 .{ }^{1,2}$ The transition from Debye relaxation to stretched-exponential relaxation (SER) marks the onset of the glass transformation range. The transformation is complete when, by convention, the relaxation time, $\tau$, increases to the experimental time scale of $100-1000 \mathrm{~s}$, corresponding to the glass transition temperature, $T_{\mathrm{g}}$. In the glass transformation range, $\tau$ often increases faster than Arrhenius, and is well approximated by the Vogel-Fulcher-Tammann (VFT) law, $\tau$ $=\tau_{0} \exp \left(A /\left(T-T_{0}\right)\right){ }^{1}$

$\beta$ and $\tau$ are therefore two fundamental parameters that describe a liquid in the glass transformation range. A challenge for a theory of the glass transition is to propose a description of these parameters. $\beta$ and $\tau$ have been discussed in a number of popular theoretical approaches. ${ }^{1-6}$ A notable feature of these approaches is that $\beta$ and $\tau$ are often treated separately. One group of theories has offered the mechanism for the increase of $\tau$, and includes the Adam-Gibbs entropy theory, ${ }^{3}$ free volume theory, ${ }^{4}$ elastic models, and other approaches (for a recent review, see ref 1). Another group of theories has derived $\beta$ for SER (see, e.g., refs 2, 5, and 6).

In view of this, it remains unclear what the relationship between $\beta$ and $\tau$ is, or if one exists at all. On the other hand,

$\dagger$ University of Cambridge.

$\doteqdot$ Naval Research Laboratory.

$\S$ George Mason University. because the increase of nonexponentiality and relaxation time are the two signatures of glass transformation, it is natural to ask if there exists a fundamental process of slowing down of molecular motion that affects both quantities. If a single mechanism affects both $\beta$ and $\tau$, it should be reflected in a welldefined relationship between these parameters at different temperatures in the glass transformation range.

In this paper, we show that a common relationship between $\beta$ and $\tau$ exists for all temperatures in the glass transformation range: $\log (\tau)$ is an approximately linear function of $1 / \beta$, followed by a crossover to a higher linear slope. We discuss the observed behavior in the elastic picture of the glass transition. In this picture, the key to the observed common relationship between $\beta$ and $\tau$ is that the two quantities are governed by the same parameter, the liquid elasticity length, $d_{\mathrm{el}}$. The increase of $d_{\mathrm{el}}$ on lowering the temperature increases $\tau$ and decreases $\beta$, resulting in the observed common relationship between $\beta$ and $\tau$. In this approach, we also discuss the crossovers of $\beta$ and $\tau$ at low temperature.

\section{Experimental Results for the Relationship between $\beta$ and} $\tau$

We have analyzed the experimental data on dielectric relaxation, including our recent results, as well as earlier data. ${ }^{7-23}$ At each temperature, $\beta$ and $\tau$ were determined from the location and width of the dielectric loss peak, respectively. Prompted by our recent work on the glass transition, ${ }^{24-26}$ we have plotted $\log (\tau)$ as a function of $1 / \beta$. In Figure 1 , this dependence is shown for 15 different glass-forming systems.

Figure 1 immediately highlights our central point, namely, that a common relationship, $f(\beta, \tau)=0$, can be identified for the studied systems in the entire range of glass transformation. First, at high temperature, $\log (\tau)$ is approximately proportional to $1 / \beta$. Second, as the temperature is reduced, a crossover to another higher slope takes place.

Generally, presenting the data as in Figure 1 is attractive, since it does not require scaling by $T_{\mathrm{g}}$, an arbitrary quantity from a theoretical point of view. In addition to providing a universal relationship between $\beta$ and $\tau$, the plot in Figure 1 can 

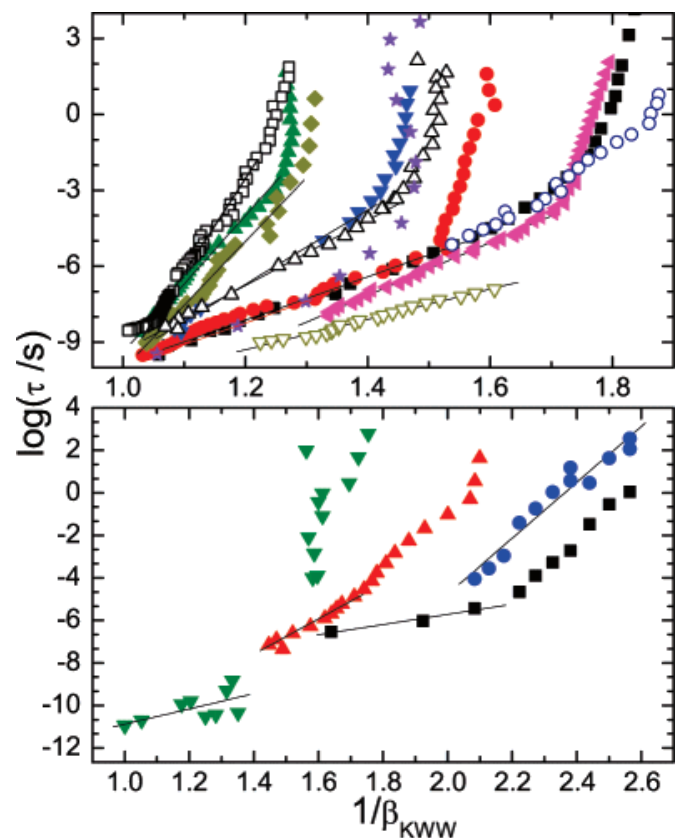

Figure 1. $\log (\tau)$ vs $1 / \beta$ for 15 glass-forming systems. Upper panel:

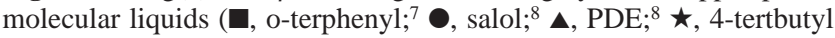
pyridine $;{ }^{9} \nabla, 54 \%$ chlorinated biphenyl $; ;^{10} \downarrow$, propylene carbonate ${ }^{8}$ leftpointing triangle, dibutylphthalate; ${ }^{11} \square, \mathrm{KDE} ;{ }^{12} \mathrm{O}, \mathrm{BMMPC} ;{ }^{12} \triangle, 62 \%$ chlorinate biphenyl: ${ }^{13} \nabla$, diisobutylphthalate ${ }^{14}$ ). Lower panel: $\boldsymbol{\Delta}$, polyvinylacetate; ${ }^{12} \boldsymbol{O}$, polycyclohexylmethacrylate $;{ }^{15} \mathbf{\square}$, polyvinylethylene; ${ }^{16} \boldsymbol{\nabla}$, bromopentane. ${ }^{17}$ Lines are guides for the eye. These represent the materials for which data are available covering a broad range and exhibiting "normal" behavior (i.e., $\beta$ decreases with decreasing temperature). There are some glass-formers (e.g., NMEC, ${ }^{18}$ MTHF, ${ }^{19}$ 1,4-polyisoprene, ${ }^{20}$ polymethylphensiloxane, ${ }^{21} 1$-propanol, ${ }^{8}$ and etha$\mathrm{nol}^{8}$ ) for which $\beta$ is almost constant over the supercooled regime, and correspondingly there is a weak or absent crossover. In other materials, a neighboring dispersion, such as the normal mode (e.g., polyoxybutylene ${ }^{22}$ ) or a secondary peak (BMPC $\left.{ }^{12}\right)$, obfuscates accurate analysis of the $\alpha$-process. For propylene glycol and glycerol, ${ }^{11} \log (\tau)$ changes with $\beta$ but does not show the linear behavior as in this figure.

serve as a convenient comparative representation of nonexponentiality in different systems approaching their glass transition.

We note that the pattern in Figure 1 is extended to the domain of high pressure. In the dielectric spectroscopy experiment, the increase of $\tau$ at higher pressure can be counterbalanced by an increase of temperature. For many systems, it has been shown ${ }^{27}$ that different combinations of pressure and temperature that keep $\tau$ constant always give the same value of $\beta$. This signifies the universality of the relationship between $\beta$ and $\tau$.

We also note that for the present materials there is a breakdown of the general correlation found between $\beta$ at $T_{\mathrm{g}}$ and the derivative of $\log (\tau)$ with respect to $T_{\mathrm{g}} / T$ at $T_{\mathrm{g}}$, fragility. ${ }^{28}$ However, this correlation is known to be only approximate, with various exceptions having been reported. ${ }^{29,30}$

There is no a priori reason why the location of the dielectric loss peak, $\tau$, should be correlated with its width, $\beta$. Hence, the observed common relationship between $\beta$ and $\tau$ in many glassforming liquids, as shown in Figure 1, is unexpected and striking. Its existence strongly suggests that SER and the VFT law reflect the same slowing-down mechanism operative in the glass transformation range.

Figure 1 presents a challenge for a theory of the glass transition. First, a theory should identify the slowing-down mechanism in a liquid that gives both SER and the VFT law. The origin of the observed common relationship between $\beta$ and $\tau$ can then be sought in the dependence of $\beta$ and $\tau$ on some parameter that describes the slowing-down mechanism. Finally, a theory should explain the crossover to a higher slope, clearly observed in Figure 1.

In order to rationalize the observed behavior, one requires an approach to the glass transition that offers descriptions of both $\beta$ and $\tau$. Some of these have been proposed (see, for example, the trapping diffusion model in ref 31 ). In this paper, we discuss the observed relationship based on the recently developed approach, which considers how a stress relaxation mechanism of a liquid changes on lowering the temperature. ${ }^{24-26}$

\section{Relationship between $\boldsymbol{\beta}$ and $\boldsymbol{\tau}$ in the Elastic Theory of Glass Transition}

Glass transition is one of the most controversial problems in condensed matter physics. ${ }^{1,32}$ Many theories have been proposed to explain the universal anomalous properties of liquids in the glass transformation range. Only a small proportion of them discussed the elastic response of a liquid, whereas others proposed different parameters that control the glass transition, including volume, entropy, energy, and many others. ${ }^{1,32}$ Because a glass is different from a liquid by virtue of its ability to support shear stress, we consider that stress relaxation is the central physical property as far as the glass transition is concerned. However, no theory has been able to explain the glass transition solely on the basis of stress relaxation or elasticity of a liquid. Recently, we have proposed how to do that. We have addressed the glass transition problem by discussing elastic fields in a liquid and considering how the stress relaxation mechanism changes on lowering the temperature. ${ }^{24-26}$ Below, we review this approach and extend it in order to understand the observed relationship between $\beta$ and $\tau$.

\section{Liquid Elasticity Length and the Origin of Slow Relaxation}

Relaxation and flow in a liquid proceed by elementary localized structural rearrangements, during which atoms jump out of their cages. We call these rearrangements local relaxation events (LREs). Because the divergence of the elastic field due to a LRE is zero, it can be viewed, in a simple model, as a pure shear event. ${ }^{1}$ For this reason, we discuss the dynamics of shear LREs. A structural rearrangement that accompanies a LRE produces elastic shear stress which can propagate through the system. The important question is how does this stress affect relaxation of other LREs in the system?

Lets consider how the stresses created by remote LREs propagate to a given local relaxing region in the center. Relaxation of the central event involves deformation of the "cage" around the jumping atom, and therefore depends on the stresses that propagate from the remote LREs to the center. A remote shear LRE creates the elastic shear waves which include waves of high frequency. This is because the deformation, associated with a localized atomic jump, creates a wave with a length comparable to interatomic separations, and hence with a frequency on the order of the Debye frequency. At high $\omega>$ $1 / \tau$ frequency, a liquid supports propagating shear waves, which propagate stress and its variations from remote LREs to the central point. If $\tau$ is generally defined as the time of decay of shear stress in a liquid, ${ }^{33} d_{\mathrm{el}}=c \tau$ gives the length of this decay, where $c$ is the speed of sound. Here, $d_{\mathrm{el}}$ gives an estimation of the maximal range over which shear stress decays in a liquid. At the microscopic level, the relevance of $d_{\mathrm{el}}=c \tau$ is as follows. A high-frequency shear wave from a LRE propagates stress until a remote LRE takes place at the front of the wave, at which point the wave front is absorbed by the remote LRE. Suppose 
this happens at distance $d_{\mathrm{el}}$ from the original LRE. $d_{\mathrm{el}}$ can be calculated from the condition of equality of the travel time, $d_{\mathrm{el}} /$ $c$, and the time at which the remote LRE takes place at point $d_{\mathrm{el}}$. This latter time is given by $\tau$, because $\tau$ is microscopically defined as the average time between two consecutive LREs, and we obtain $d_{\mathrm{el}}=c \tau$ as before.

$d_{\mathrm{el}}$, therefore, defines the maximal distance over which the central LRE is affected by elastic stresses due to other LREs in a liquid. For this reason, $d_{\mathrm{el}}$ can be called the liquid elasticity length. Note that because it takes time $\tau$ for the central event to relax, its relaxation is affected by all stresses that have enough time to propagate to the center, i.e., those located distance $c \tau$ away (after time $\tau$, the central event relaxes, and the process repeats). In this sense, the definition $d_{\mathrm{el}}=c \tau$ is self-consistent.

To discuss how $d_{\text {el }}$ governs relaxation in more detail, lets consider the dynamics of LREs in a liquid perturbed by an external field. Some time ago, Orowan's introduced the terms "concordant" and "discordant" events: ${ }^{34}$ a concordant local rearrangement is accompanied by a strain in the direction agreeing with the applied external stress, and reduces the energy and local stress. A discordant rearrangement, on the other hand, increases the energy and local stress. This has led to a general result that stress relaxation by earlier concordant events leads to the increase of stress on later relaxing regions in a system. Goldstein applied the same argument to a viscous liquid: ${ }^{35}$ consider a system under external stress which is counterbalanced by stresses supported by local regions. After a concordant LRE, a local region supports less stress after the event than before; therefore, other local regions in the system should support more stress after that event than before. ${ }^{35}$

Let $\Delta p$ be the increase of shear stress on a current LRE due to previous concordant LREs. If $n$ is the current number of LREs, $\Delta p$ is a monotonically increasing function of $n$. The increase of stress on a currently relaxing region increases its activation barrier, $V$. It has been argued that $V$ is given by the elastic shear energy of a surrounding liquid. ${ }^{36}$ This result was confirmed by the experimental data, showing that the activation barrier increases with the shear energy. ${ }^{36}$ Because, as discussed by Orowan and Goldstein, previous LREs reduce stress in the direction "concordant" to the external stress, the increase of shear stress on later rearranging regions consistently increases the shear strain on them in the same direction, increasing the shear energy and therefore $V$. The increase of $V, \Delta V$, due to the additional stress, $\Delta p$, is the work, $\int \Delta p \mathrm{~d} q$. If $q_{\mathrm{a}}$ is the characteristic volume, ${ }^{36} \Delta V=\Delta p q_{\mathrm{a}}$, and we find $V=V_{0}+$ $q_{\mathrm{a}} \Delta p$, where $V_{0}$ is the high-temperature activation barrier. Because $\Delta p$ is a monotonically increasing function of $n$ and $V$ $=V_{0}+q_{\mathrm{a}} \Delta p$, we find that $V$ is also a monotonically increasing function of $n$. This provides the elastic feed-forward interaction mechanism between LREs. ${ }^{24}$

To quantify this discussion further, we consider relaxation, induced in a liquid by a pulse of an external field. At time zero, stresses supported by local regions counterbalance macroscopic stress. As relaxation proceeds, each LRE reduces stress locally until the macroscopic stress is relaxed by a certain finite number of LREs and the liquid comes to equilibrium. At times smaller than $L / c$, where $L$ is the system size, external stress can be considered constant, and the stress redistribution argument of Orowan-Goldstein applies. Alternatively, we can consider an external field constantly compensating for the decreases of local stresses. In the resulting steady flow, $\tau$ is a relaxation time due to an increment of external perturbation, and can be viewed as the time of the liquid's retardation behind an external field. Lets introduce time-dependent variable $n(t)$, the current number of

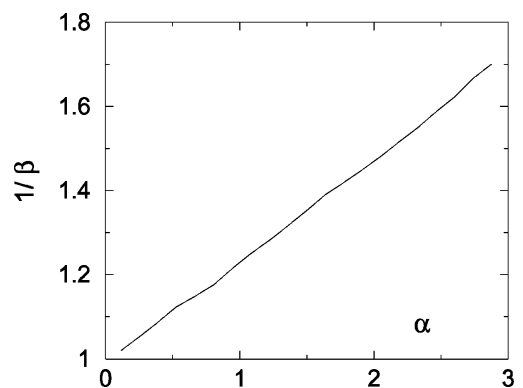

Figure 2. $1 / \beta$ as a function of $\alpha$, obtained from the solution of eq 2 .

relaxing events induced by an increment of external stress in the sphere of radius $d_{\mathrm{el}} . n(t)$ starts from zero and increases to its final value $n_{\mathrm{r}}$. Lets consider the current LRE to relax to be in the center of the sphere of radius $d_{\mathrm{el}}$. As discussed above, all previous remote concordant LREs within distance $d_{\mathrm{el}}$ from the center participate in the elastic feed-forward interaction, increasing stress $\Delta p$ on the central region and hence increasing $V$ for the central LRE. Note that all stresses within a distance of $d_{\mathrm{el}}$ $=c \tau$ have enough time to propagate to the center and affect the relaxation of the central event (recall the self-consistency in the definition of $d_{\mathrm{el}}$ ).

$\Delta p$ can be calculated by integrating the contributions of remote concordant LREs. This, together with $V(n)=V_{0}+q_{\mathrm{a}} \Delta p$ from above, gives ${ }^{24}$

$$
\begin{gathered}
V(n)=V_{0}+V_{1} \frac{n}{n_{\mathrm{r}}} \\
V_{1}=\pi / 2 \rho_{\mathrm{r}} q_{\mathrm{a}} \Delta p_{0} d_{0}^{3} \ln \left(2 d_{\mathrm{el}} / d_{0}\right)
\end{gathered}
$$

where $d_{0}$ is on the order of the size of a relaxing region, $\rho_{\mathrm{r}}$ is the density of $n_{\mathrm{r}}$ local relaxing regions, and $\Delta p_{0}$ is the decrease of stress due to a remote concordant LRE.

In eq $1, V$ depends on temperature through $d_{\mathrm{el}}$. It is easy to show that using $d_{\mathrm{el}}=c \tau$ and $\tau=\tau_{0} \exp (V / k T)$ in eq 1 gives the VFT law for $V$ and $\tau .{ }^{26}$ In this picture, the super-Arrhenius behavior is related to the increase of $d_{e l}$ on lowering the temperature: as the temperature is lowered and $\tau$ increases, more LREs are involved in the elastic interaction with a given LRE, increasing its activation barrier.

\section{Relationship between $\beta$ and $\tau$}

We are now set to write the equation that relates $\beta$ and $\tau$. The rate of LREs, $\mathrm{d} n / \mathrm{d} t$, is the product of the number of unrelaxed events, $\left(n_{\mathrm{r}}-n\right)$, and the event probability, $\rho=$ $\exp (V / k T)$. Since $V$ depends on $n$ (see eq 1$), \rho$ depends on $n$ as well. Introducing $q=n / n_{\mathrm{r}}$ and reduced time $t / \tau_{0}$, we write

$$
\frac{\mathrm{d} q}{\mathrm{~d} t}=(1-q) \exp \left(-\frac{V_{0}+V_{1} q}{k T}\right)
$$

Equation 2 has two parameters, $V_{0} / k T$ and $\alpha=V_{1} / k T$. We have recently shown ${ }^{24}$ that its solution is well approximated by the two-parameter SER, $q(t)=1-\exp \left(-(t / \tau)^{\beta}\right)$. We note that, whereas $\tau$ depends on both $V_{0} / k T$ and $\alpha, \beta$ depends on $\alpha$ only; the smaller $\alpha$, the larger $\beta$ ( $\alpha=0$ gives exponential relaxation, $\beta=1$ ).

To find the relationship between $\beta$ and $\tau$, we solve eq 2 for different values of $\alpha$, fit the solution to the form of SER above, and find that $1 / \beta=1+C \alpha$, where $C$ is a constant (see Figure 2 ). Joining this result with $V_{1} \propto \ln \left(d_{\mathrm{el}}\right) \propto \ln (\tau)$ (see eq 1 ), we find that $\ln (\tau)$ is a quasi-linear function of $1 / \beta$. 


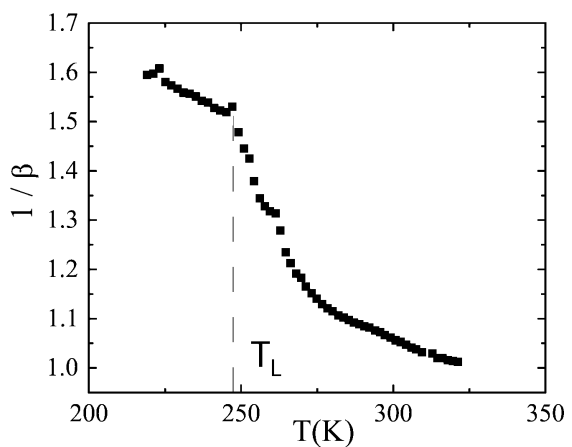

Figure 3. Temperature dependence of $1 / \beta$ in salol. ${ }^{7,8} T_{L}$ is the temperature of the crossover when $d_{\mathrm{el}}=L$.

In this picture, the key to the common relationship between $\beta$ and $\tau$ is that the two quantities are governed by the same parameter, $d_{\mathrm{el}}$. As we have seen above, the increase of $d_{\mathrm{el}}$ on lowering the temperature decreases $\beta$ because it increases $V_{1}$ (see eqs 1 and 2), and at the same time, it increases $\tau$ because the total activation barrier increases (see eq 1). This gives the universal relationship between $\beta$ and $\tau$, consistent with that observed in the experiment.

To discuss the crossover to the second higher slope in Figure 1 , we note that our theory predicts the crossovers ${ }^{24-26}$ of both $\tau$ and $\beta$ at $d_{\mathrm{el}}=L$, where $L$ is the system size. Recall that $V \propto$ $\ln \left(d_{\mathrm{el}}\right)$ (see eq 1). As long as $d_{\mathrm{el}}<L, V$ increases with temperature, because $d_{\mathrm{el}}$ increases. When $d_{\mathrm{el}} \geq L$, the system crosses over from being partially to wholly elastic, and $V$ becomes temperature-independent, $V \propto \ln (L)$ (see eq 1). When $d_{\mathrm{el}} \geq L$, further decrease of temperature has a weaker effect on $V$ related to, e.g., a density increase, but not to the increase of $d_{\mathrm{e} \text {. }}$. Therefore, this picture predicts the dynamic crossover, at which the VFT dependence changes to either Arrhenius form or another VFT dependence with smaller curvature. This is consistent with experimental results. ${ }^{8}$

The same reasoning applies to the crossover of $\beta . V_{1}$ increases on lowering the temperature because $V_{1} \propto \ln \left(d_{\mathrm{el}}\right)$ in eq 1 . This remains true as long as, on lowering the temperature, $d_{\mathrm{el}}<L$. When $d_{\mathrm{el}} \geq L, V_{1} \propto \ln (L)$ and is temperature-independent. Hence, at $d_{\mathrm{el}}=L, V_{1}$ shows a kink. Consequently, because $\beta$ decreases with $V_{1} / k T$ (see Figure 2), we find that $d_{\mathrm{el}}=L$ should mark the crossover of $\beta$ to a lower slope. Taking salol as an example, we observe the well-defined kink at low temperature and the crossover to the lower slope (see Figure 3), consistent with the theory.

Consistent with this picture, we find that the crossovers in Figure 1 correspond to temperatures at which both $\tau$ and $\beta$ show a crossover. At the crossover temperature, we find that $\tau$ crosses over from one VFT dependence to another. Therefore, each linear behavior in Figure 1 can be assigned to a single VFT dependence. At about the same temperature, $\beta$ shows a crossover as well. For example, for salol, the kink of $\beta$ takes place at about $1 / \beta=1.5$ (see Figure 3), the same value at which the crossover in Figure 1 is seen.

We also find that the crossover of $\tau$ is less pronounced as compared with that of $\beta$. As a result, the detection of the crossover of $\tau$ requires derivative analysis, ${ }^{8}$ whereas the crossover of $\beta$ is readily seen in Figure 3. The reason for this is explained in our theory as follows. $V$ and $\tau$ are defined by the sum of a constant term $V_{0}$ and a temperature-dependent term $V_{1}$ (see eq 1), whereas $\beta$ is solely defined by $\alpha=V_{1} / k T$ (see eq 2). Hence, $\beta$ is more sensitive to the crossover at $d_{\mathrm{el}}=L$ as compared to $\tau$. As a result, the crossovers seen in Figure 1 are due, to a large extent, to the crossover of $\beta$ : for salol, the pronounced crossover to the lower slope in Figure 3 gives the crossover to the higher slope in Figure 1.

Therefore, we find that the predictions from our theoretical approach are consistent with the crossovers seen in Figure 1. To quantify this discussion further, we make two observations. First, from the definition of $d_{\mathrm{el}}$, the relaxation time at the crossover is $\tau=L / c$. If a typical experimental value of $L$ is taken in the range of, for example, $1-10 \mathrm{~mm}$, and of $c$ as 1000 $\mathrm{m} / \mathrm{s}, \tau$ at the crossover is $10^{-6}-10^{-5} \mathrm{~s}$. This is in a reasonable agreement with the crossovers seen in Figure 1, at which $\tau$ is in the $10^{-6}-10^{-4} \mathrm{~s}$ range. Second, we can evaluate the crossover temperature as follows. Multiplying $\tau=\tau_{0} \exp \left(A /\left(T-T_{0}\right)\right)$ by $c$, we find $d_{\mathrm{el}}=a \exp \left(A /\left(T-T_{0}\right)\right)$. Here, $\tau_{0}$ is the Debye oscillation period, $a$ is an interatomic separation of about $1 \AA$, and we have taken into account that $a \approx c \tau_{0}$. Take, for example, salol, for which $A=839 \mathrm{~K}$ and $T_{0}=195 \mathrm{~K} \cdot{ }^{23}$ Using $d_{\mathrm{el}}=L$ at the crossover and $L$ in the $1-10 \mathrm{~mm}$ range as above, we find the crossover temperature, $T_{L}$, in the range $241-247 \mathrm{~K}$. This agrees reasonably well with the crossover temperature seen in Figure 3, as well as with the crossover temperature of $\tau .^{23}$

We note that, for some liquids, the condition $d_{\mathrm{el}} \geq L$ can hold true even at high temperature. ${ }^{26}$ In this case, no crossover is observed on lowering the temperature, consistent with some of our experimental results. However, our picture predicts that the dynamic crossover at $d_{\mathrm{el}}=L$ would be observed in these liquids if the measurements were extended to higher temperature. This temperature should be high enough so that $d_{\mathrm{el}}$ reduces below $L$ in order to observe the crossover at lower temperature. We also note that the increase of $V$ on lowering the temperature can have a contribution related to the increase of density. This contribution comes in addition to the increase of $d_{\mathrm{el}}$ and does not depend on $d_{\mathrm{el}}$ or $L$. Hence, the crossover at $d_{\mathrm{el}}=L$ can be reduced depending on the relative weight of the contributions that arise from the increase of $d_{\mathrm{el}}$ and density.

By comparing theoretical and experimental results, we have found that the insights from our theory offer a consistent way to understand and interpret the experimental data in Figure 1, including the crossovers. At the same time, we note that our theory also predicts that, at low temperature when $d_{\mathrm{el}} \geq L$, viscosity can weakly depend on the size of a macroscopic system (with the caveats discussed above). Whereas system size effects were observed in nanoconfinement and thin films, no such dependence has been reported for a macroscopic system, except in our recent experiment. ${ }^{25}$ It is interesting to study this effect in other systems and in more detail. We hope that our discussion can stimulate further experimental work.

\section{Summary}

In summary, we have shown that the nonexponentiality parameter, $\beta$, and relaxation time, $\tau$, are commonly related in the glass transformation range: $\log (\tau)$ is an approximately linear function of $1 / \beta$, followed in some cases by a crossover to a higher linear slope. We rationalized this relationship using the elastic theory of the glass transition. In this picture, the key to the common observed relationship between $\beta$ and $\tau$ is that the two quantities are governed by the same parameter, the liquid elasticity length, $d_{\mathrm{el}}$. The increase of $d_{\mathrm{el}}$ on lowering the temperature increases $\tau$ and decreases $\beta$, resulting in the observed relationship. In this approach, we also discussed the crossovers of $\beta$ and $\tau$ at low temperature.

Acknowledgment. We are grateful to Prof. R. Böhmer and E. Röessler for providing their data in digital form, to Prof. V. 
V. Brazhkin for discussions, and to EPSRC and the Office of Naval Research for support.

\section{References and Notes}

(1) Dyre, J. C. Rev. Mod. Phys. 2006, 78, 953.

(2) Phillips, J. C. Rep. Prog. Phys. 1996, 59, 1133

(3) Adam, G.; Gibbs, J. H. J. Chem. Phys. 1965, 43, 139.

(4) Cohen, M. H.; Turnbull, D. J. Chem. Phys. 1959, 31, 1164.

(5) Palmer, R. G.; Stein, D. L.; Abrahams, E.; Anderson, P. W. Phys. Rev. Lett. 1984, 53, 958. L463.

(6) Dominics, C. D.; Orland, H.; Lainee, F. J. Phys., Lett. 1985, 46,

(7) León, C.; Ngai, K. L. J. Phys. Chem. B 1999, 103, 4045 2043.

(8) Stickel, F.; Fischer, E. W.; Richert, R. J. Chem. Phys. 1996, 104,

(9) Blochowicz, T. et al. J. Chem. Phys. 2006, 124, 134503.

(10) Casalini, R.; Santangelo, P. G.; Roland, C. M. J. Phys. Chem. B 2002, 106, 11492

(11) Dixon, P. K.; Wu, L.; Nagel, S. R.; Williams, B. D.; Carini, J. P. Phys. Rev. Lett. 1990, 65, 1108.

(12) Stickel, F. Ph.D. thesis, Mainz University, Germany (Shaker, Aachen), 1995.

(13) Casalini, R.; Paluch, M.; Fontanella, J. J.; Roland, C. M. J. Chem. Phys. 2002, 117, 4901.

(14) Sekula, M.; et al. J. Phys. Chem. B 2004, 108, 4997.

(15) Roland, C. M.; Casalini, R. Macromolecules, in press.

(16) Roland, C. M.; et al. Macromolecules 2003, 36, 4954

(17) Berberian, J. G.; Cole, R. H. J. Chem. Phys. 1986, 84, 6921.
(19) Qi, F.; et al. J. Chem. Phys. 2003, 118, 7431

(20) Roland, C. M.; Schroeder, M. J.; Fontanella, J. J.; Ngai, K. L. Macromolecules 2004, 37, 2630.

(21) Paluch, M.; Roland, C. M.; Pawlus, S. J. Chem. Phys. 2002, 116 10932.

(22) Casalini, R.; Roland, C. M. Macromolecules 2005, 38, 1779.

(23) Casalini, R.; Ngai K. L.; Roland, C. M. Phys. Rev. B 2003, 68, 014201 .

(24) Trachenko, K. Phys. Rev. B 2007, 75, 212201.

(25) Trachenko, K.; Brazhkin, V. V. J. Phys.: Condens. Matter 2008 20,075103 .

(26) Trachenko, K. arXiv:cond-mat/0704.2975v1.

(27) Ngai, K. L.; Casalini, R.; Capaccioli, S.; Paluch, M.; Roland, C. M. J. Phys. Chem. B 2005, 109, 17356.

(28) Böhmer, R.; Ngai, K. L.; Angell, C. A.; Plazek, D. J. J. Chem. Phys. 1993, 99, 4201.

(29) Roland, C. M.; Paluch, M.; Rzoska, S. J. J. Chem. Phys. 2003 , $119,12439$.

(30) Roland, C. M.; Hensel-Bielowka, S.; Paluch, M.; Casalini, R. Rep Prog. Phys. 2005, 68, 1405.

(31) Odagaki, T.; Matsui, J.; Hiwatari, Y. Phys. Rev. E 1994, 49, 3150.

(32) Langer, J. Phys. Today 2007, 60, 8.

(33) Landau, L. D.; Lifshitz, E. M. Theory of Elasticity; Pergamon Press: 1986.

(34) Orowan, E. Proceedings of the First National Congress of Applied Mechanics; American Society of Mechanical Engineers: New York, 1952; p 453.

(35) Goldstein, M. J. Chem. Phys. 1969, 51, 3728.

(36) Dyre, J. C.; Olsen, N. B.; Christensen, T. Phys. Rev. B 1996, 53, (1) 